\title{
Humanoid robot RH-1 for collaborative tasks: a control architecture for human-robot cooperation
}

\author{
Concepción A. Monje*, Paolo Pierro and Carlos Balaguer \\ University Carlos III of Madrid, Avd. Universidad 30, 28911 Leganés, Madrid, Spain
}

(Received 29 September 2008; final version received 19 January 2009)

\begin{abstract}
The full-scale humanoid robot RH-1 has been totally developed in the University Carlos III of Madrid. In this paper we present an advanced control system for this robot so that it can perform tasks in cooperation with humans. The collaborative tasks are carried out in a semi-autonomous way and are intended to be put into operation in real working environments where humans and robots should share the same space. Before presenting the control strategy, the kinematic model and a simplified dynamic model of the robot are presented. All the models and algorithms are verified by several simulations and experimental results.
\end{abstract}

Keywords: humanoid robot RH-1; modelling and control; human-robot collaborative tasks; virtual reality model

\section{Introduction}

In real life, the use of robots in typical human environments could be really profitable, especially in work tasks where robots can perform in collaboration with human agents. In order to achieve a successful collaboration, many researchers (Green et al. 2008) have studied the modalities of the interaction between the two agents (i.e. dialogue, joint solution of problems, etc.) Within the field of human-robot collaboration, we can select different kinds of interaction, as graphically summarised in Figure 1. The human can interact with the other agent (that can be a robot or also a simulator) via keyboard, a PDA system or a joystick, or even by direct contact. The robot can execute the collaboration getting information through cameras and force/torque sensors.

Human-robot collaboration has a common framework with respect to the human-robot interaction and it is important that the robot partner perceives human intentions or goals in order to achieve a common objective (Hinds et al. 2004). In order to reason human intentions during a collaborative task (such as a collaborative transportation or assembly), the sensorial system of the robot should be integrated. The information coming from this system becomes an input to the control algorithm, which allows to carry out the collaborative task while maintaining the whole system stable. The control algorithms proposed in this paper have been tested using the model of the humanoid robot RH-1 (Figure 2), a prototype totally developed within the research team Robotics Lab in the University Carlos III of Madrid.
The RH-1 is a robot $1.50 \mathrm{~m}$ tall and $50 \mathrm{~kg}$ in weight with 21 degrees of freedom (DOF). The DOF are distributed as follows: 6 for each leg, 4 for each arm and 1 for the chest. The electronic hardware (together with the batteries) is embedded in the robot: two main computers PC-104 which are used for controlling the servomotors and for high-level interaction (image and sound processing). The communication system works on a CANBUS fieldbus. The robot is also equipped with a camera, microphones and speakers in order to facilitate cooperation with humans. For stability control, inertial sensors are needed: accelerometers and gyroscopes are also embedded in the system. The system can be operated from a laptop or a workstation using wireless communication.

The paper is organised as follows. Section 2 shows the control architecture proposed, in which both posture stability and collaborative control are aimed. The posture stability controls are presented in Section 3, in which a simplified model of the dynamics of the $\mathrm{RH}-1$ robot is given, based on the model of the single inverted pendulum. Section 4 deals with the collaborative control scheme, proposing a kinematic model of the arms and a joint manipulation scheme. Finally, some conclusions and future works are drawn in Section 5.

\section{The control architecture}

The novel control scheme proposed in this paper is the one shown in Figure 3, where two different control loops are

${ }^{*}$ Corresponding author. Email: cmonje@ing.uc3m.es 


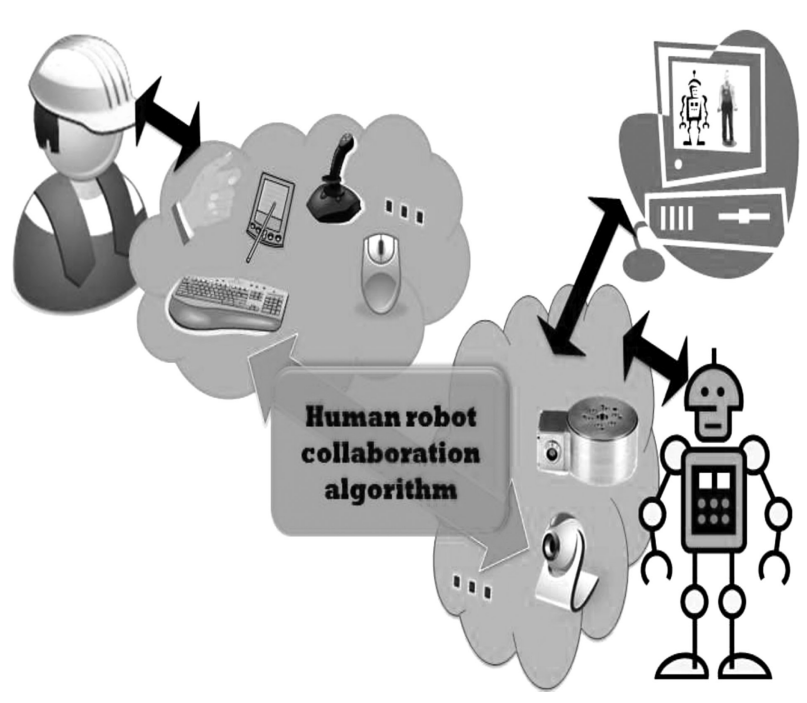

Figure 1. Modalities of the human-robot collaboration.

considered: (a) humanoid's body posture stability and (b) collaborative control loop.

Given a task, there are different patterns for the arms and the legs in order to generate a stable posture for the robot. The main function of the collaborative control loop is to ensure that these motion patterns are stable and achievable by the robot during the collaborative task. The

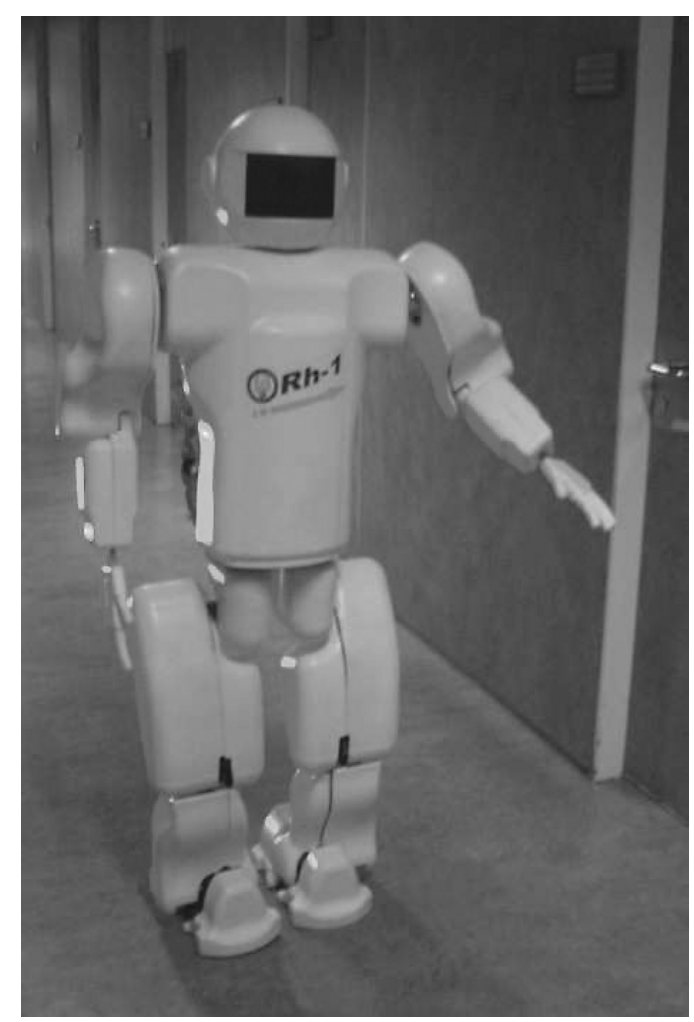

Figure 2. Humanoid robot RH-1.

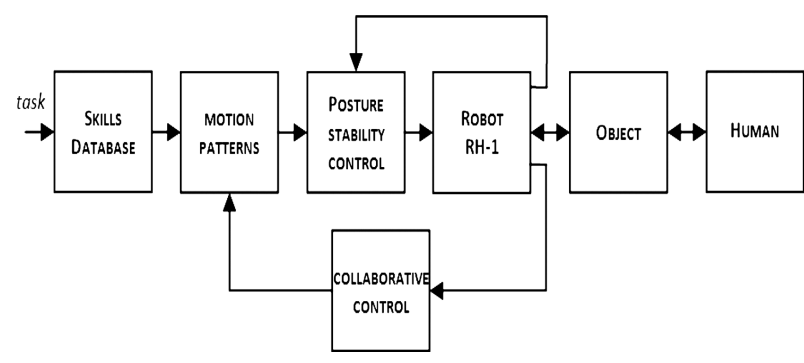

Figure 3. General control architecture.

kinematic model of the robot is needed for that purpose. In an inner loop, a posture control is needed to guarantee the stability of the humanoid for the different postures determined by the motion patterns. In this case, the dynamic model of the robot must be used. The posture must be controlled in realtime through the information from the encoders of the servomotors, since the forces caused by the object and the man during the activity may undermine the stability of the system. Several models can be used in order to achieve the stability. For instance, it is possible to evaluate the Zero Moment Point (ZMP) and correct the posture on-line using the force/torque sensors in the feet. Possible ways of correcting the posture are also the compensation of the waist position and the adjustment of the leg joints. Eventually, it is also possible to regulate the position of the feet (in case of rough terrain or inevitable errors in the position of the feet) and the gait velocity (Kajira et al. 2006. Kim et al. 2008). The measurement of the ZMP can also be important for the quasi on-line estimation of the evolution of the ZMP. If the intentions of the master are known in advance, a correct pattern can be chosen for the arms and the legs. Therefore, using, for instance, space and time prediction, it is possible to calculate the next probable ZMP position and also select a better walking pattern. A possible approach, proposed in Fernández et al. (2001), is based on an active human-robot cooperation system based on intention recognition, using the Hidden Markov Model. Anyway, these solutions produce great algorithm complexities, which makes their use very difficult in real situations.

\section{Posture stability control}

\subsection{The model of the single inverted pendulum}

In a very simplified way, the dynamic model of the humanoid robot RH-1 can be considered similar to that of the inverted pendulum in Figure 4.

The similarity is established under the following assumptions. The mass of the humanoid $(m)$ is concentrated at its mass centre (tip of the pendulum), which is at a distance $l$ from the floor. The mass of the rigid link is then considered negligible. Besides, the action (torque $T$ ) that allows the mass $m$ to move a specific angle $\theta$ at a 


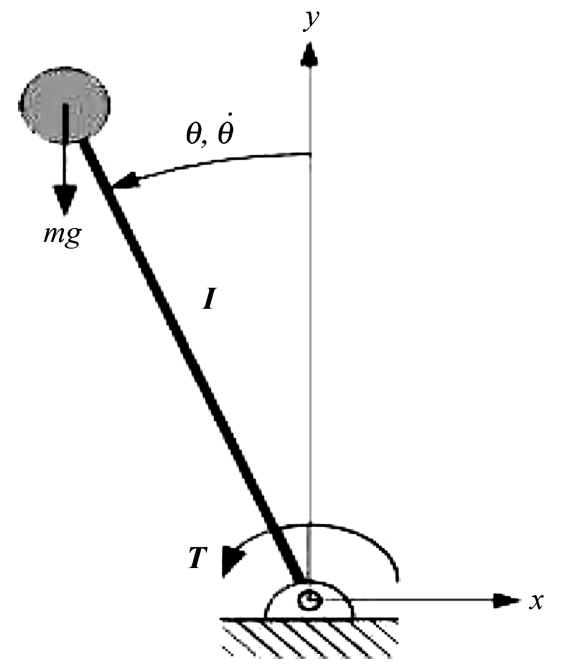

Figure 4. Single inverted pendulum.

speed $\dot{\theta}$ (movement of the mass centre during the walking action) is effected by a servomotor (ankle of the humanoid robot) fixed at the end of the link (floor). This servomotor performs the control action to ensure the stability of the system during the walking action.

It is clear that this model is not complex enough to model the whole dynamics of the humanoid robot and to consider its non-linearities. However, as can be checked in the recent literature, it gives very good results, even experimentally, as a first approximation. For instance, in Kim et al. (2008), Bachelier et al. (2008), Kumar et al. (2008) the inverted pendulum model is used to study the stability and the dynamic walking problems in humanoids.

To write the equation of motion of the pendulum (Khalil 1999), let us identify the forces acting on the tip. There is a downward gravitational force equal to $m g$, where $g$ is the acceleration due to gravity. There is also a frictional force resisting the motion, which we assume to be proportional to the speed of the tip with a coefficient of friction $k$.

Using Newton's second law of motion, we can write the equation of motion in the tangential direction as

$$
m l \ddot{\theta}=-m g \sin \theta-k l \dot{\theta} .
$$

Writing the equation of motion in the tangential direction has the advantage that the link tension, which is in the normal direction, does not appear in the equation. To obtain a state model for the pendulum, let us take the state variables as $x_{1}=\theta$ and $x_{2}=\dot{\theta}$. Then, the state equations are

$$
\begin{aligned}
& \dot{x_{1}}=x_{2} \\
& \dot{x_{2}}=-\frac{g}{l} \sin x_{1}-\frac{k}{m} x_{2} .
\end{aligned}
$$

To find the equilibrium points, we set $\dot{x_{1}}=\dot{x_{2}}=0$ and solve for $x_{1}$ and $x_{2}$ as

$$
\begin{aligned}
& 0=x_{2} \\
& 0=-\frac{g}{l} \sin x_{1}-\frac{k}{m} x_{2} .
\end{aligned}
$$

The equilibrium points are located at $(n \pi, 0)$, for $n=$ $0, \pm 1, \pm 2, \ldots$ From the physical description of the pendulum, it is clear that the pendulum has only two equilibrium positions corresponding to the equilibrium points $(0,0)$ and $(\pi, 0)$. Other equilibrium points are repetitions of these two positions, which correspond to the number of full swings the pendulum would make before it rests at one of the two equilibrium positions.

Physically, we can see that these two positions are quite distinct from each other. While the pendulum can indeed rest at the $(0,0)$ equilibrium point, it can hardly maintain at the $(\pi, 0)$ point because infinitesimally small disturbance from that equilibrium will take the pendulum away. The difference between the two equilibrium points is in their stability properties.

Another version of the pendulum equation arises if we can apply a torque $T$ to the pendulum. This torque is viewed in our case as a control input in the following equation

$$
\begin{aligned}
& \dot{x_{1}}=x_{2} \\
& \dot{x_{2}}=-\frac{g}{l} \sin x_{1}-\frac{k}{m} x_{2}+\frac{1}{m l^{2}} T .
\end{aligned}
$$

\subsection{The posture control problem}

The scheme for the control of the inverted pendulum is the one in Figure 5.

The purpose is to control the position of the mass of the pendulum $\theta$ through the action of a servomotor $M(s)$ that gives the appropriate torque at each moment in order to follow the position reference $\theta_{\text {ref }}$.

In our case, the servomotor of the ankle (and the rest of joints) of the humanoid RH-1 is already controlled experimentally in closed loop by an auto-tuning driver so that the output position of the tip $\theta$ follows a reference $\theta_{\text {ref }}$ given by the walking pattern. However, it must be said that the whole system works in open loop, that is, there is not an on-line feedback of the position of the mass in order to compensate for disturbances. That is, the trajectory is loaded (off-line) in the driver and then the servomotor follows the reference

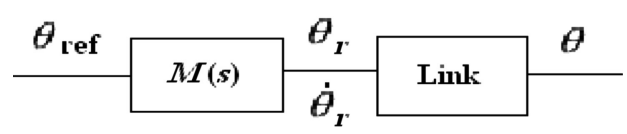

Figure 5. Posture control system. 


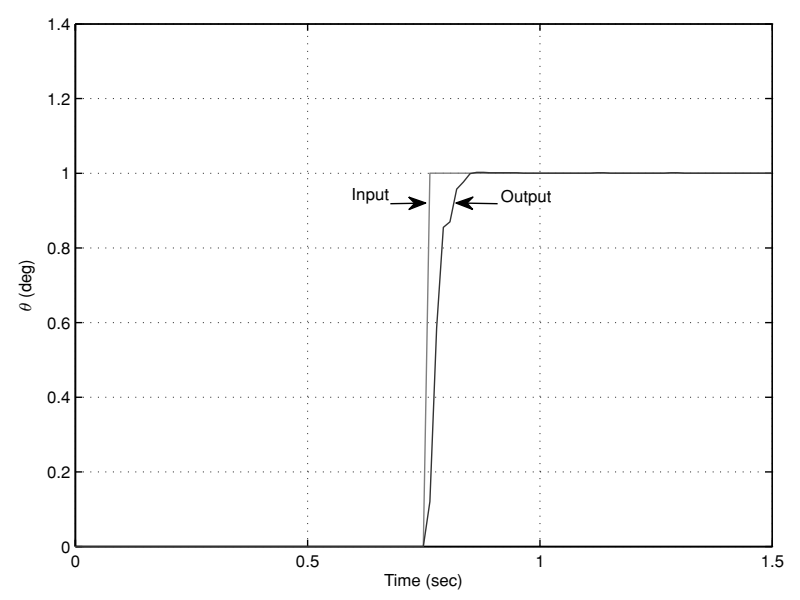

Figure 6. Experimental input and output signals for the servomotor identification.

with a negligible tracking error due to the internal closed loop tuned by the auto-tuner.

Our aim here is to obtain a model that represents the dynamics achieved in practice, using the model of the single inverted pendulum. For that purpose, the model of the servomotor in closed loop, $M(s)$, must be identified to take it as the reference system for the whole system in open loop. That is, as shown in the block diagram in Figure 5, $\left(\theta-\theta_{r}\right) \rightarrow 0$ and $\left(\dot{\theta}-\dot{\theta}_{r}\right) \rightarrow 0$.

\subsection{Identification of the servomotor}

The model of the servomotor in closed loop has been identified through the experimental input and output signals generated in the identification test, which are represented (only half a cycle) in Figure 6.

By these two signals and considering a linear parametric model of third order for the motor, the resulting transfer function is

$$
M(s)=\frac{55.03 s^{2}+5439 s+2.73 \cdot 10^{6}}{s^{3}+111.2 s^{2}+5.14 \cdot 10^{4} s+2.73 \cdot 10^{6}} .
$$

\subsection{Model matching technique}

Once the servomotor in closed loop is identified, the control problem in open loop must be solved so that the output of the link $\theta$ follows the reference $\theta_{\text {ref }}$ (see Figure 5). Besides, in our scheme $\left(\theta_{r}-\theta_{\text {ref }}\right) \rightarrow 0$, since the motor associated to the link is able to follow the reference with a negligible error. It means that, somehow, the dynamics of the link is cancelled in this control purpose. In fact, we are looking for the control action $(T)$ that allows this fact. To achieve this, the model matching technique described in Isidori (1995) is used, based on the input-output linearisation of the system. The equations obtained from the application of the technique are the ones presented here.

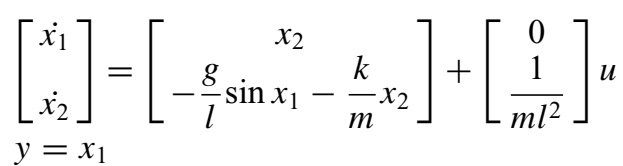

Therefore, the direct relation between the input and output of the system is given by

$$
\ddot{y}=-\frac{g}{l} \sin x_{1}-\frac{k}{m} x_{2}+\frac{1}{m l^{2}} u
$$

with $u=T$ and $y=\theta$. The purpose is to obtain the control law $u$ so that $y$ follows $\theta_{\text {ref }}$ as $\theta_{r}$ follows $\theta_{\text {ref }}$, that is, a control law so that the whole dynamics matches the model $M(s)=\frac{\theta_{r}}{\theta_{\text {ref }}}$ obtained previously by identification. In order to do so, we define $u$ as

$$
u=m l^{2}\left[\left(\frac{g}{l} \sin x_{1}+\frac{k}{m} x_{2}\right)+v\right]
$$

so that $\ddot{y}=v$ (from Equation 7). Choosing

$$
v=\dot{\theta_{r}}+a\left(\theta_{r}-y\right)
$$

it is obtained that

$$
\ddot{y}=\dot{\theta}_{r}+a\left(\theta_{r}-y\right) .
$$

The value of $a$ is selected in order to obtain the minimum tracking error.

\subsection{Simulation results}

The control system described in the previous section has been implemented in Simulink ${ }^{\circledR}$ considering the model of the single inverted pendulum and the model $M(s)$ of the servomotor in closed loop (used as reference model). The parameters of the system are $m=50 \mathrm{~kg}, l=1 \mathrm{~m}$, $g=9.8 \mathrm{~m} / \mathrm{sec}^{2}, k=0.1$, and $a=0.1$. In Figure 7 the angular position and velocity of the tip are represented for a signal reference of value $\pi$. As can be observed, the control strategy allows the tip to keep in the equilibrium point $(\pi, 0)$.

Once this first approach has been achieved, the efforts are currently devoted to the control of the system in closed loop so that it can be robust to disturbances and model mismatches. 


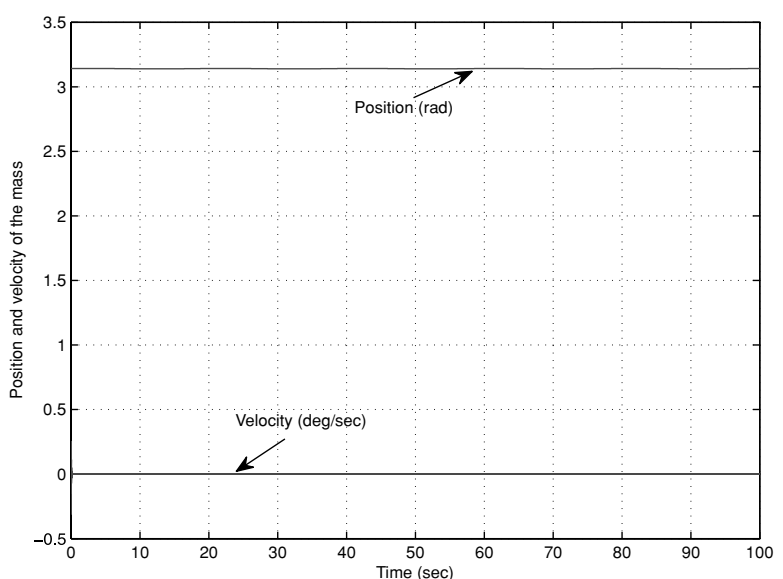

Figure 7. Angular position and velocity of the tip.

\subsection{Experimental results}

This section shows the experimental results obtained when the robot takes a complete step in open loop. Figure 8 shows the different joints of the humanoid robot. Figures 9 to 18 show the walking patterns and the outputs of the joints involved in the walking action, that is, $q_{1}$ : right ankle (roll), $q_{2}$ : right ankle (pitch), $q_{3}:$ right knee (pitch), $q_{4}:$ right hip (pitch), $q_{5}$ : right hip (roll), $q_{8}$ : left hip (roll), $q_{9}$ : left hip (pitch), $q_{10}$ : left knee (pitch), $q_{11}$ : left ankle (pitch), $q_{12}$ : left ankle (roll). It must be taken into account that these measurements in radians must be divided by 160 in the case of $q_{1}$ and $q_{12}$ and by $4 \times 160$ in the case of the rest of joints in order to obtain the position of each link, due to the gear reduction. From these figures it is concluded that the robot can take a step in open loop.

\section{Collaborative control}

For the collaborative task, a prior aspect to be ensured is that the humanoid robot is able to jointly manipulate the object
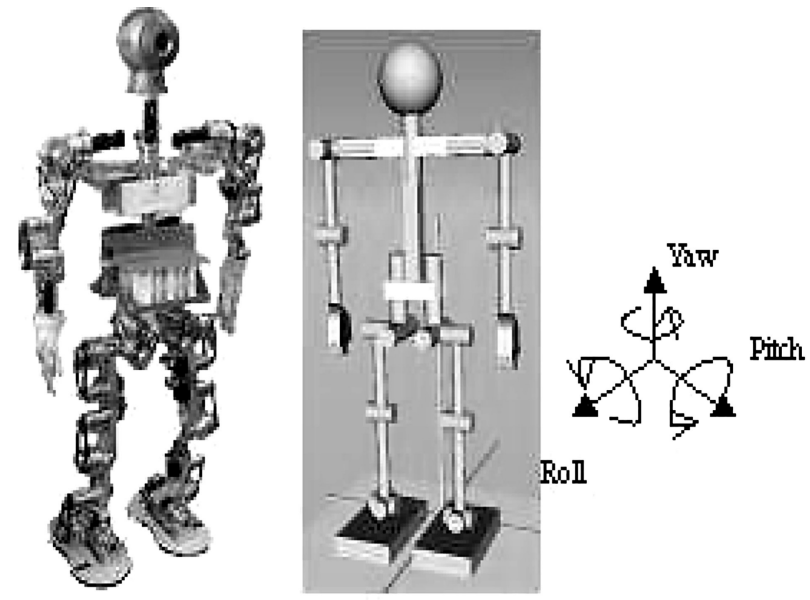

Figure 8. Scheme of the joints.

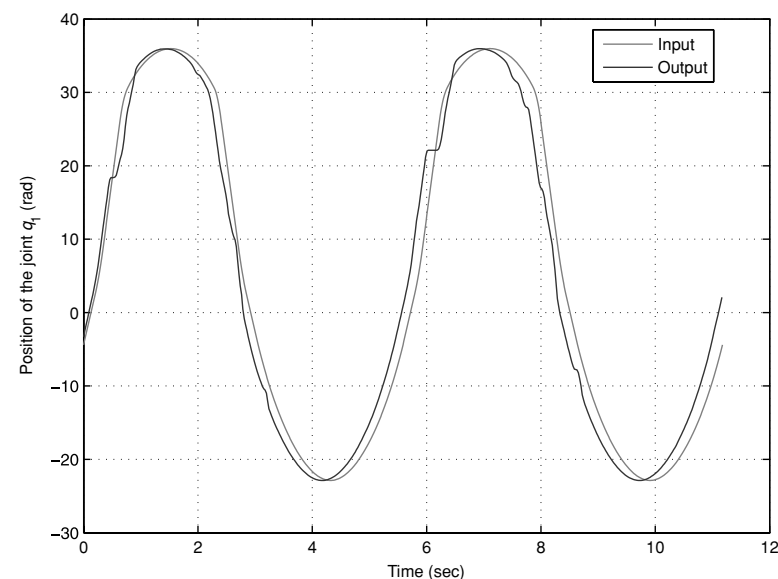

Figure 9. Joint $q_{1}$ : right ankle (roll).

with the human. The joint manipulation can be studied modelling the robot arms and the object as a closed chain.

\subsection{Model of the robot's arms}

While for open-chain manipulators the classical problem is to calculate the joint angles (and motor torques) for a given trajectory for the end-effector, for closed-chain manipulators the solution must be calculated considering as a main constraint that the object to manipulate must be supported. In fact, as it is shown in Figure 19, which represents the arms of the humanoid robot RH-1 in a schematic way, the closed chain has been cut in the middle of the bar, which becomes the end-effector. The whole kinematics will be solved calculating the kinematics of each arm and then imposing the following conditions for the common end effector

- same position with respect to the origin

- same orientation with respect to the origin.

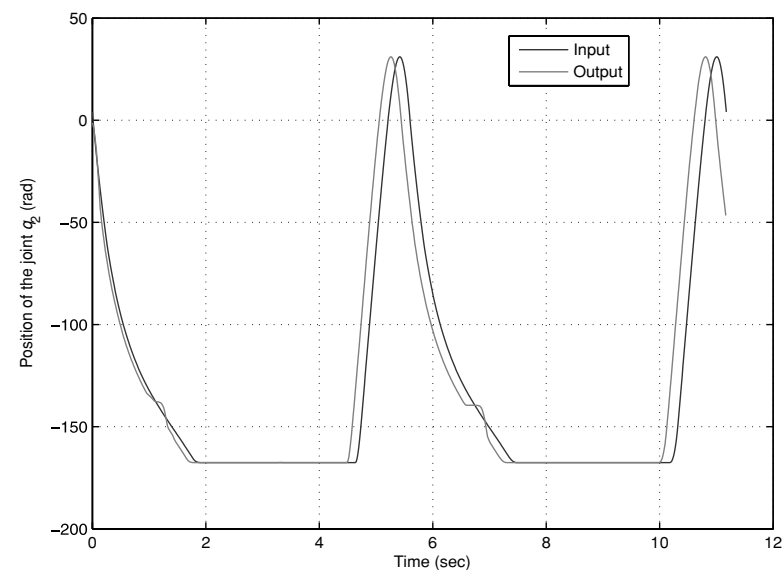

Figure 10. Joint $q_{2}$ : right ankle (pitch). 


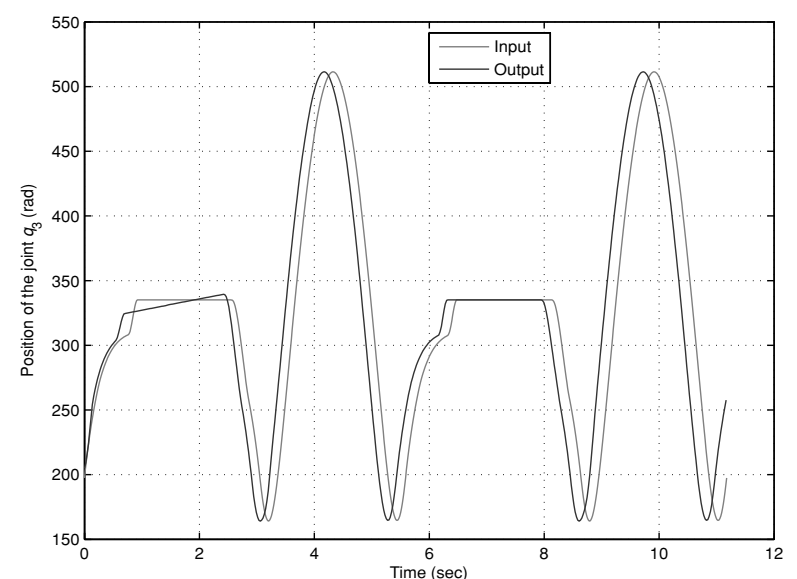

Figure 11. Joint $q_{3}$ : right knee (pitch).

Obviously, the mechanical limitations reduce all these possibilities. The robot humanoid considered has $9 \mathrm{DOF}$, which would not be sufficient for specifying the $12 \mathrm{DOF}$ required for carrying out the chain and specifying position and orientation of the end effector.

\subsection{Inverse kinematics of the robot's arm}

The proposed solution presented here only specifies the position (without the orientation) of the end effector. The remaining 6 DOF are necessary to hold the object. Nakamura et al. (1987) introduced the concept of task priority in relation to the inverse kinematic problem of redundant robot manipulators. Using the pseudoinverse of the Jacobian matrix, it is possible to determine the joint angles of the robot for executing a task, while accomplishing one or several lower-priority subtasks (Sciavicco et al. 2000).

Letting $\mathbf{x}_{\mathbf{r}}$ and $\mathbf{x}_{\mathbf{l}}$ be the position and orientation of the right and left arms, respectively, the solutions $\mathbf{q r}_{\mathbf{r}}$ and $\mathbf{q}_{\mathbf{l}}$

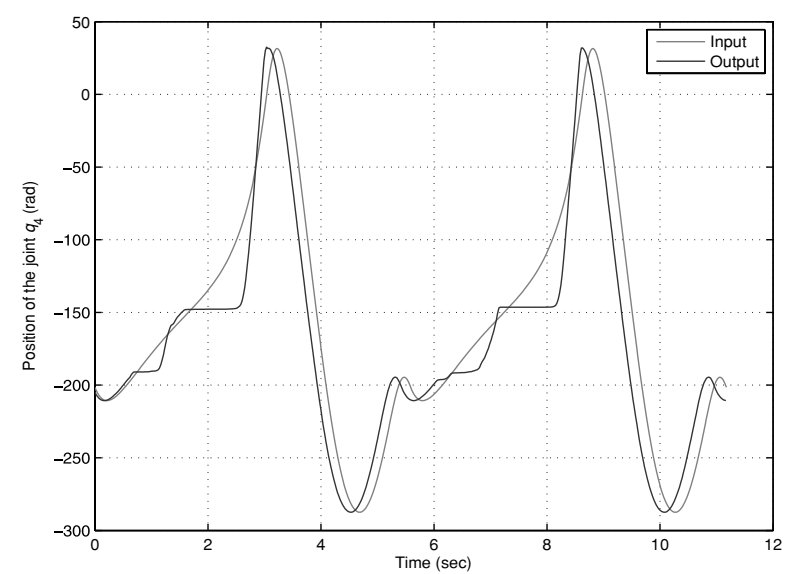

Figure 12. Joint $q_{4}$ : right hip (pitch).

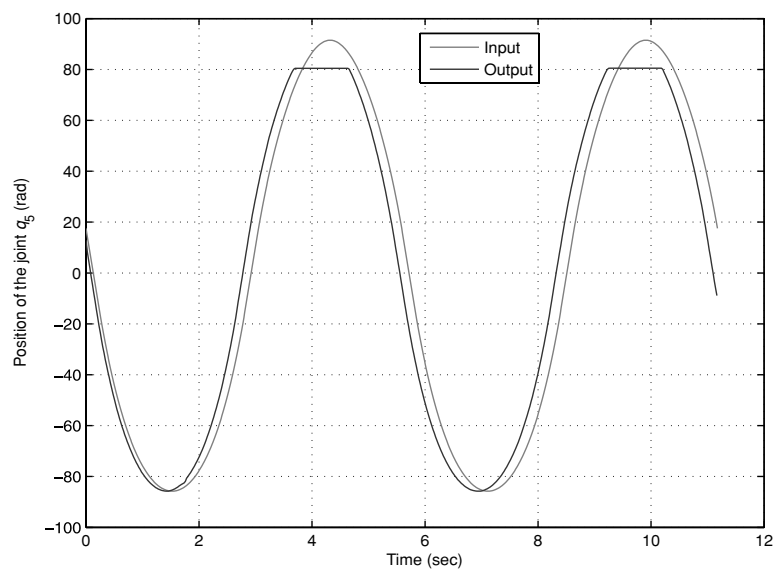

Figure 13. Joint $q_{5}$ : right hip (roll).

referring to the joint angles of the right and left arms must be found. The aims to be considered are the following ones

- the right and left arm must have the same end effector

- the end effector must follow a desired trajectory.

As stated before, the first condition is the most important and, hence, has the highest priority. This condition can be summarised as $\mathbf{x}_{\mathbf{r}}=\mathbf{x}_{\mathbf{l}}$. In an equivalent manner

$$
\dot{\mathbf{x}}_{\mathbf{r}}=\dot{\mathbf{x}}_{\mathbf{l}} \Rightarrow \mathbf{J}_{\mathbf{r}} \dot{\mathbf{q}}_{\mathbf{r}}=\mathbf{J}_{\mathbf{l}} \dot{\mathbf{q}}_{\mathbf{l}}
$$

where $\mathbf{J}_{\mathbf{r}}$ and $\mathbf{J}_{\mathbf{l}}$ are the Jacobian matrixes of the right and left arms, respectively, and $\dot{\mathbf{q}}_{\mathrm{r}}$ and $\dot{\mathbf{q}}_{\mathrm{I}}$ are the joints velocities of the two arms.

In order to find the solutions $\mathbf{q}_{\mathbf{r}}$ and $\mathbf{q}_{\mathbf{p}}$, the problem has been solved using the method of the Lagrange multipliers.

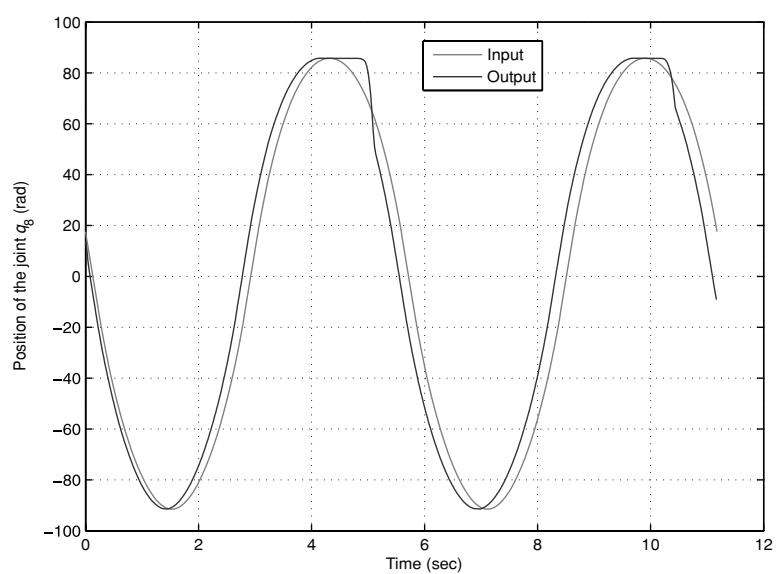

Figure 14. Joint $q_{8}$ : left hip (roll). 


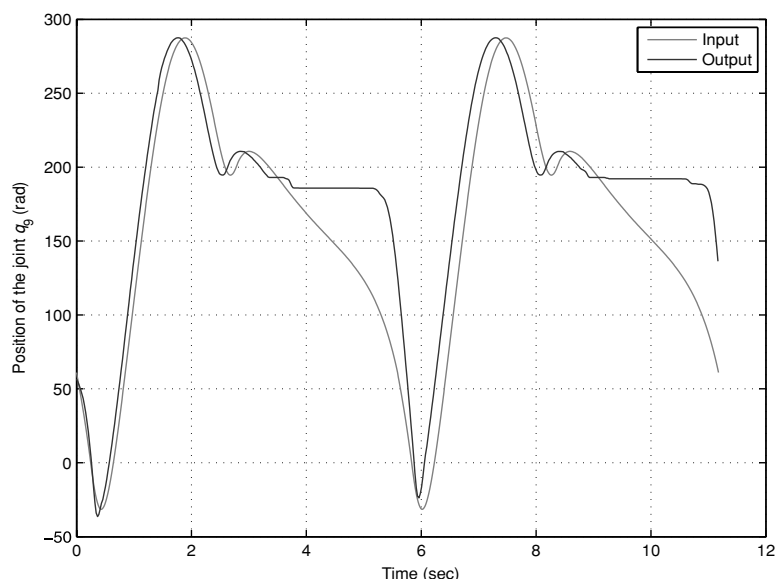

Figure 15. Joint $q_{9}$ : left hip (pitch).

Let us consider the following function

$$
g\left(\dot{\mathbf{q}}_{\mathbf{r}}\right)=\frac{1}{2}\left(\dot{\mathbf{q}}_{\mathbf{r}}-\dot{\mathbf{q}}_{\mathbf{0}}\right)^{T}\left(\dot{\mathbf{q}}_{\mathbf{r}}-\dot{\mathbf{q}}_{\mathbf{0}}\right)+\lambda\left(\mathbf{J}_{\mathbf{l}} \dot{\mathbf{q}}_{\mathbf{l}}-\mathbf{J}_{\mathbf{r}} \dot{\mathbf{q}}_{\mathbf{r}}\right)
$$

where $\lambda$ is an unknown vector of the multipliers. In order to achieve the kinematic optimisation of the cost function (12), the following equation must be fulfilled

$$
\left(\frac{\partial g\left(\dot{\mathbf{q}}_{\mathbf{r}}\right)}{\partial \dot{\mathbf{q}}_{\mathbf{r}}}\right)^{T}=0 \Rightarrow \dot{\mathbf{q}}_{\mathbf{r}}=\mathbf{J}_{\mathbf{r}}^{\mathbf{T}} \lambda+\dot{\mathbf{q}}_{\mathbf{0}}
$$

From Equations (11) and (13) it is obtained that

$$
\lambda=\left(\mathbf{J}_{\mathbf{r}} \mathbf{J}_{\mathbf{r}}^{\mathbf{T}}\right)^{-1} \mathbf{J}_{\mathbf{l}} \dot{\mathbf{q}}_{\mathbf{I}}-\left(\mathbf{J}_{\mathbf{r}} \mathbf{J}_{\mathbf{r}}^{\mathbf{T}}\right)^{-1} \mathbf{J}_{\mathbf{r}} \dot{\mathbf{q}}_{\mathbf{0}},
$$

which can be definitely written as

$$
\dot{\mathbf{q}}_{\mathbf{r}}=\mathbf{J}_{\mathbf{r}}^{\dagger} \mathbf{J}_{\mathbf{l}} \dot{\mathbf{q}}_{\mathbf{l}}+\left(\mathbf{I}-\mathbf{J}_{\mathbf{r}}^{\dagger} \mathbf{J}_{\mathbf{r}}\right) \dot{\mathbf{q}}_{\mathbf{0}} .
$$

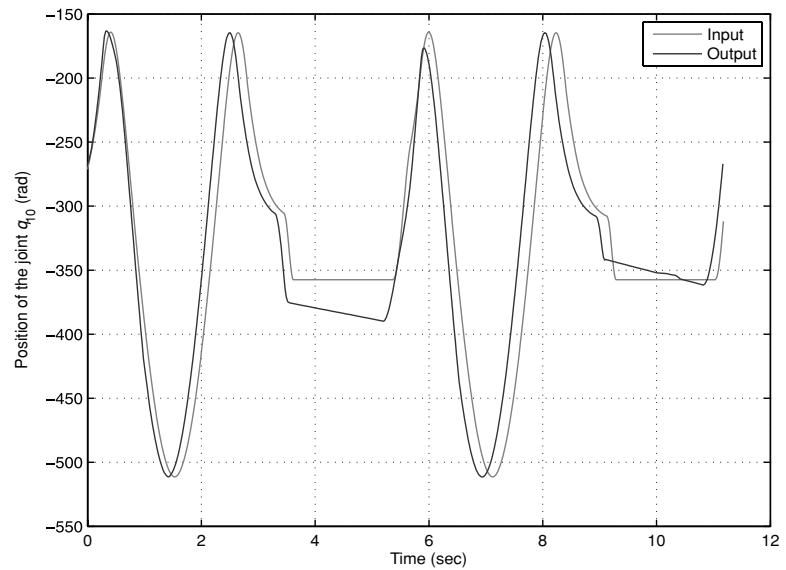

Figure 16. Joint $q_{10}$ : left knee (pitch).

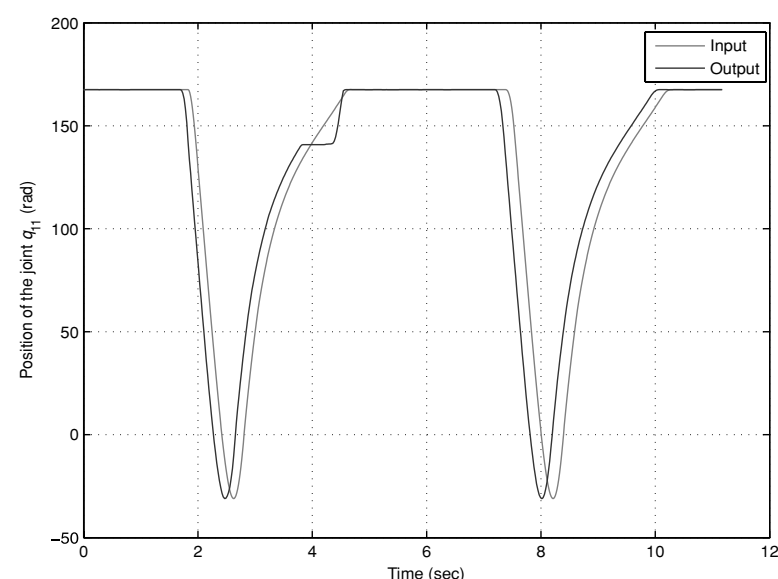

Figure 17. Joint $q_{11}$ : left ankle (pitch).

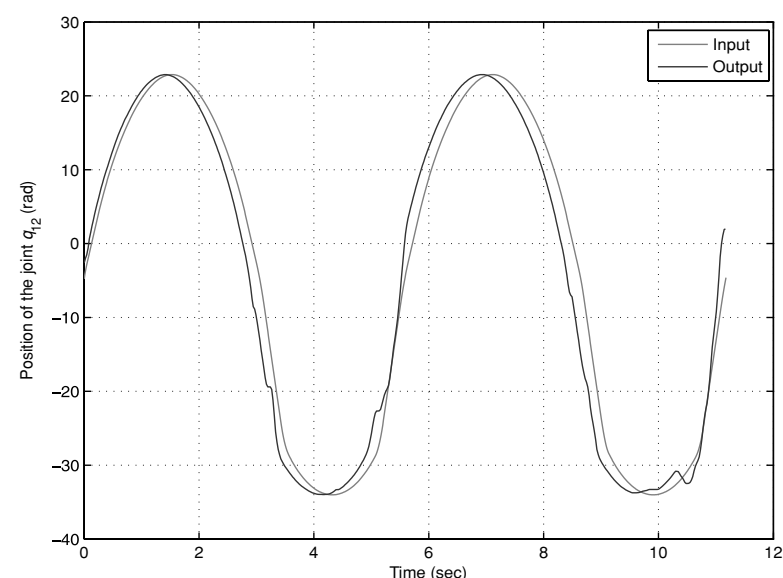

Figure 18. Joint $q_{12}$ : left ankle (roll).

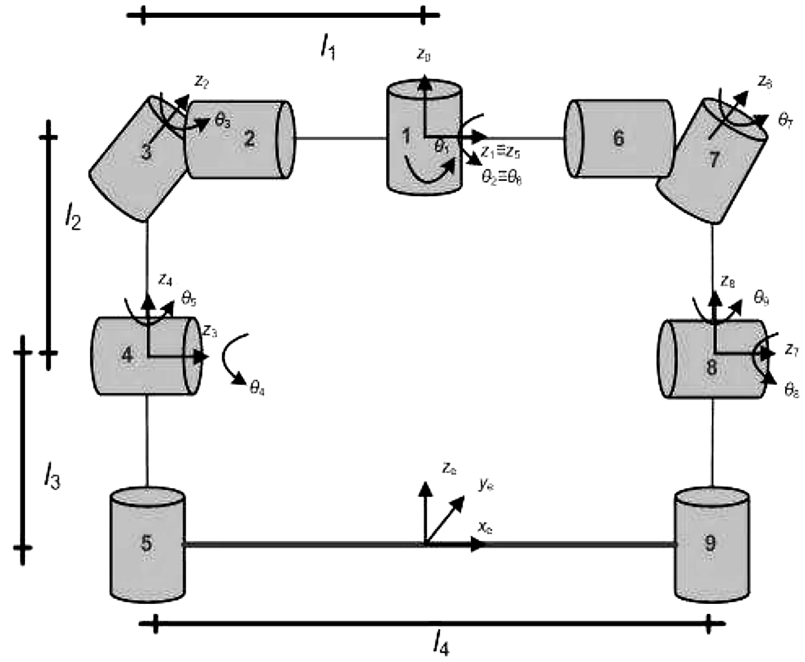

Figure 19. Kinematic model of the arms. 


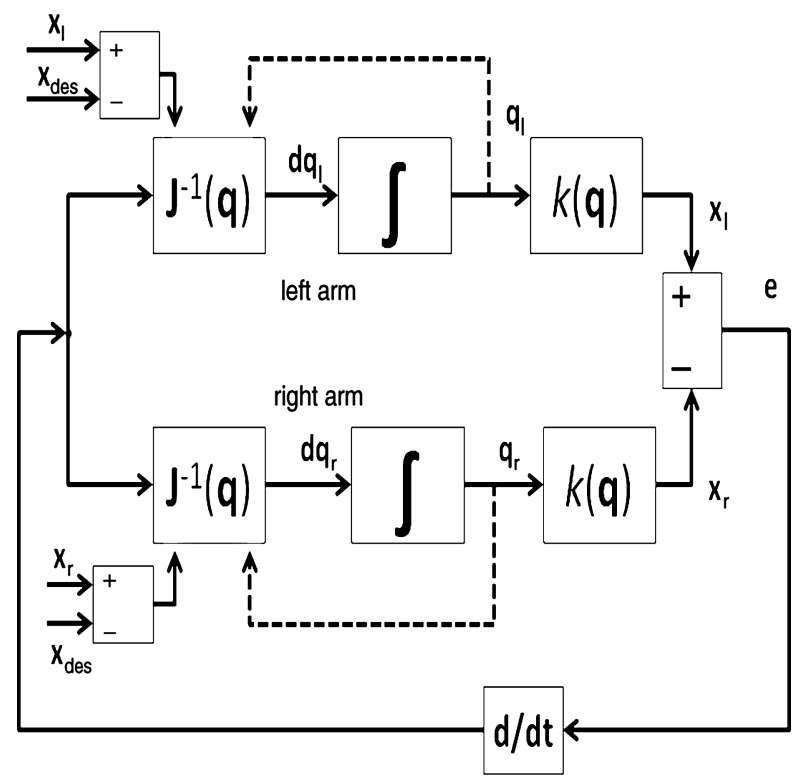

Figure 20. Inverse kinematics for the closed loop of the arms.

In this equation the notation $\mathbf{J}_{\mathbf{r}}^{\dagger}$ is used to denote the pseudo-inverse matrix of the right arm:

$$
\mathbf{J}_{\mathbf{r}}^{\dagger}=\mathbf{J}_{\mathbf{r}}^{\mathbf{T}}\left(\mathbf{J}_{\mathbf{r}} \mathbf{J}_{\mathbf{r}}^{\mathbf{T}}\right)^{-1}
$$

Equation (15) represents the solution of a closed-chain kinematics for a redundant manipulator and $\dot{\mathbf{q}}_{0}$ is a lowerpriority solution to be achieved.

The idea is to use this vector to follow the desired trajectory $\mathbf{x}_{\text {des }}$ (or its velocity $\dot{\mathbf{x}}_{\text {des }}$ ). Therefore, a possible choice could be

$$
\dot{\mathbf{q}}_{0}=k \mathbf{J}_{\mathbf{r}}^{\dagger} \dot{\mathbf{x}}_{\mathrm{des}}
$$

where $k$ is a positive constant.

The solution (15) together with the analogue solution of the left arm is represented in a schematic way in Figure 20.

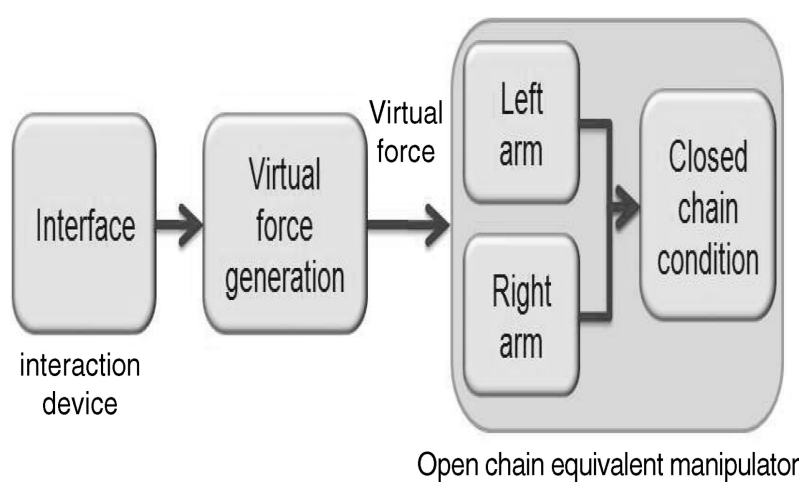

Figure 21. Joint manipulation scheme.

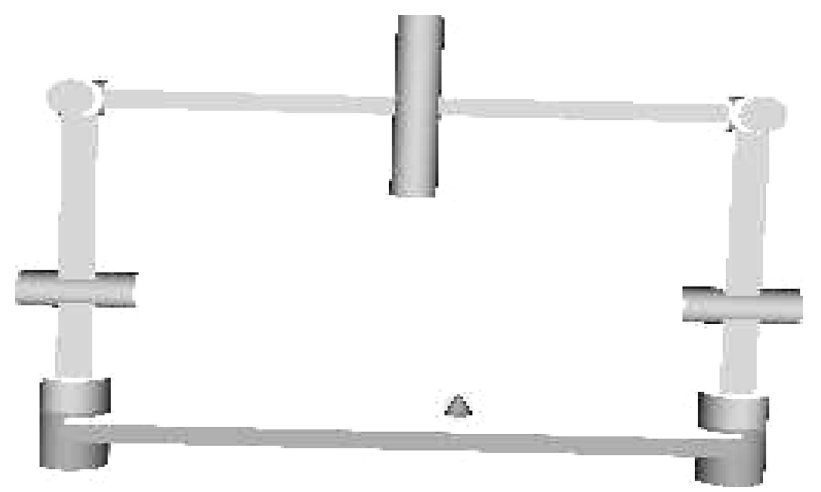

Figure 22. Virtual reality model of the arms.

\subsection{Joint manipulation}

Actually, the closed-loop manipulator becomes a manipulator which is moved through the vector $\mathbf{x}_{\text {des }}$. Hence, it can be considered equivalent to an open-loop manipulator with the end effector placed in the middle of the bar which connects the two arms of the humanoid robot RH1. Obviously, considering that, a force/position control of the end effector is possible. For that purpose the simplified scheme shown in Figure 21 is considered. The input to the manipulator can either be a real force or a virtual force generated by an external device, such as a joystick or a mouse.

\subsection{Simulation results}

The algorithm presented here has been implemented using Simulink ${ }^{\complement}$. The virtual reality model of RH-1 arms

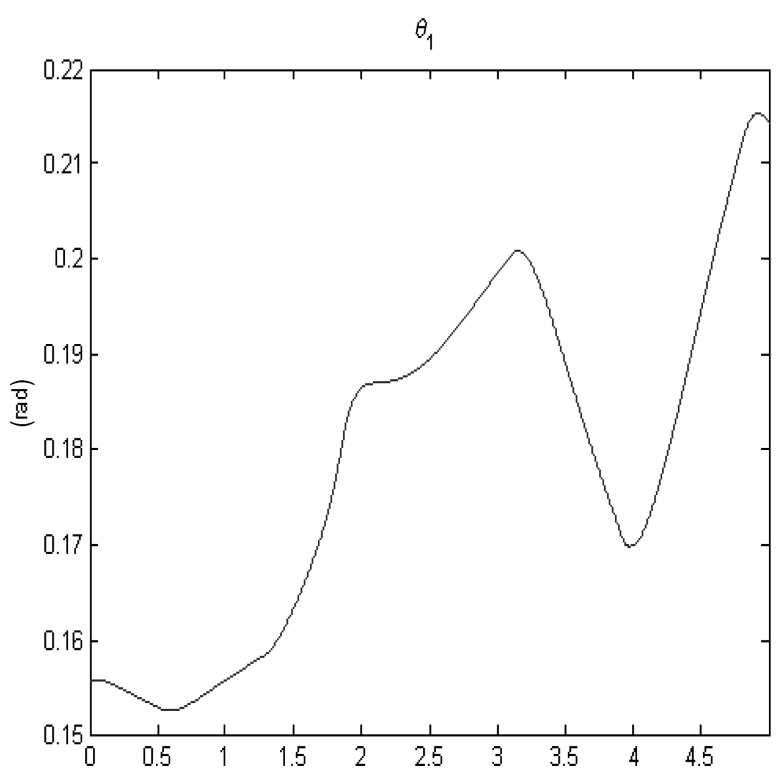

Figure 23. Joint angles of the robot's chest. 


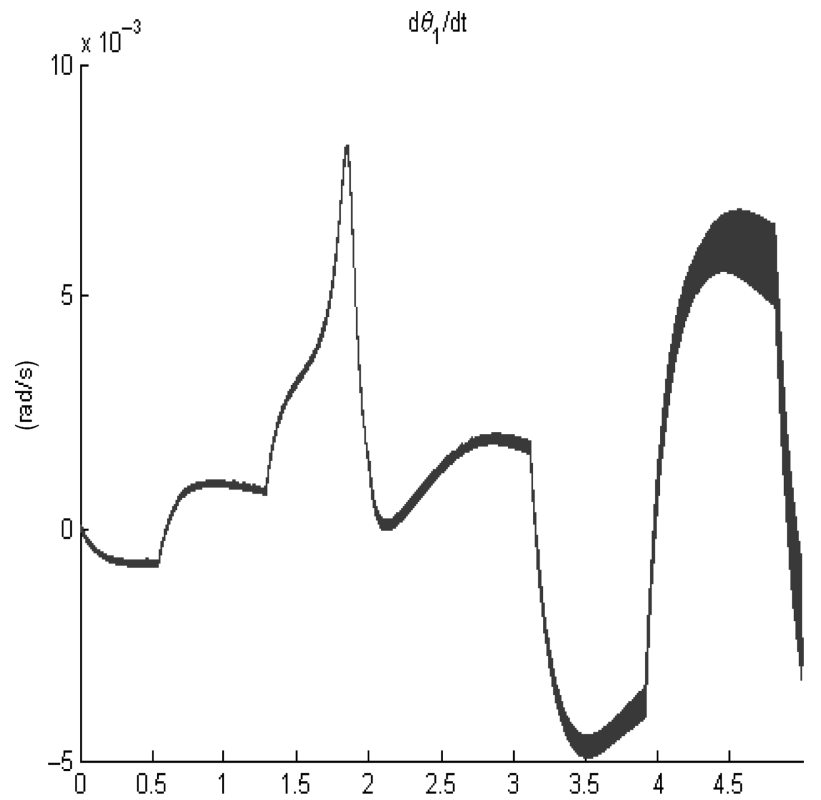

Figure 24. Joint velocities of the robot's chest.

is presented in Figure 22. A connection with the virtual world is established and a virtual force is applied with the mouse.

The algorithm has been used to test different situations and some results are presented in Figure 23 to Figure 28.

As can be observed in Figure 24, regarding the joint velocity of the robot's chest, there are several abrupt changes
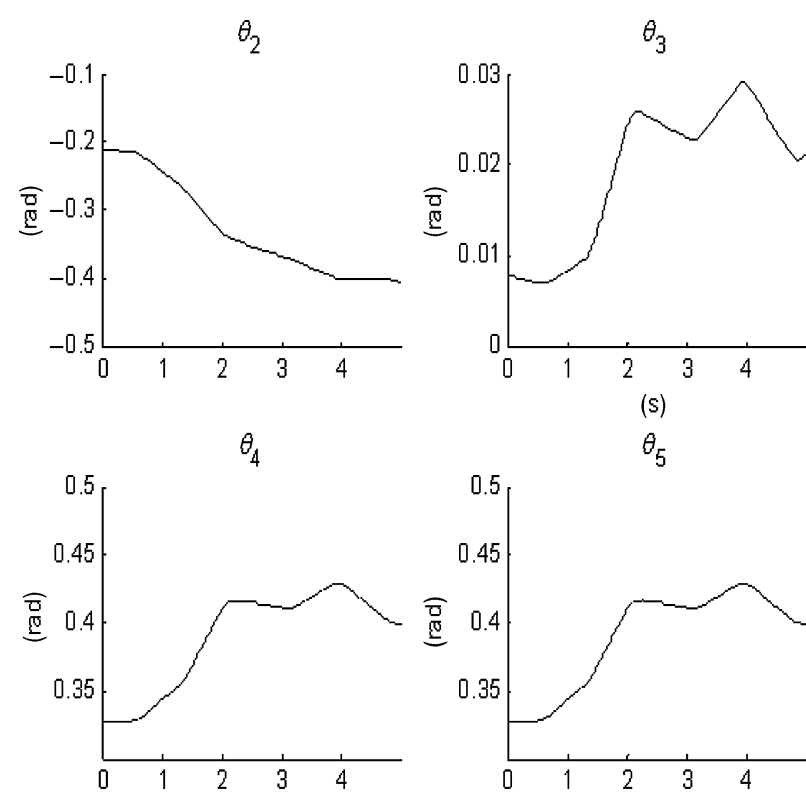

(s)
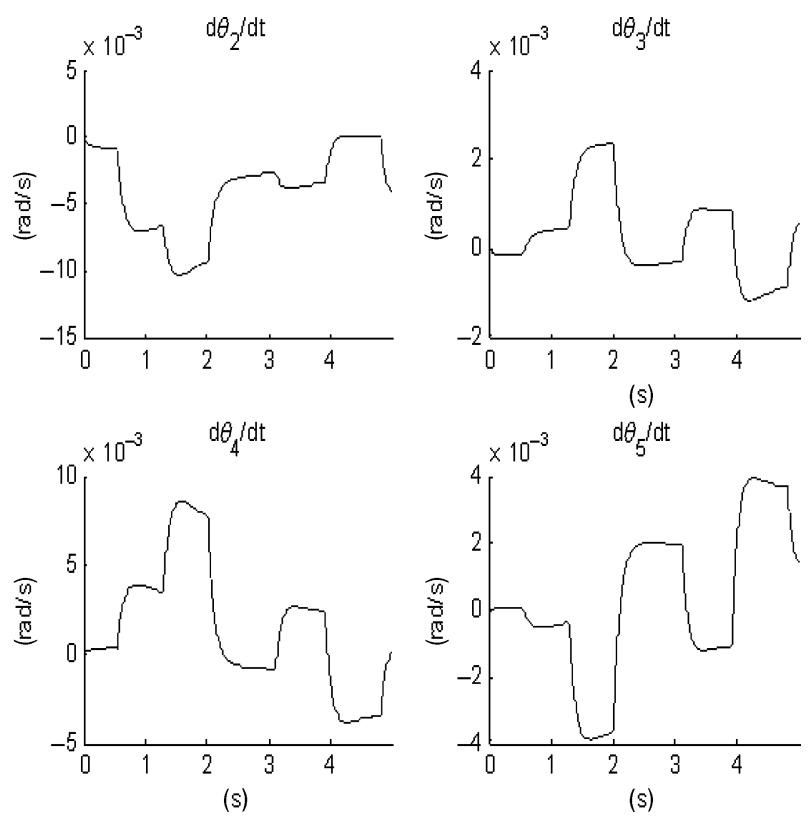

Figure 26. Joint velocities of the right arm.

which come to great peaks in the motor torques. This is due to the fact that the two manipulators work with the shared joint in the chest. Some steps are currently being taken to reduce this effect. From this study it seems clear that 9 DOF are not sufficient for the collaborative tasks. At least one yaw joint is needed in each arm and a pitch in the chest. This way the requirements for holding the object and
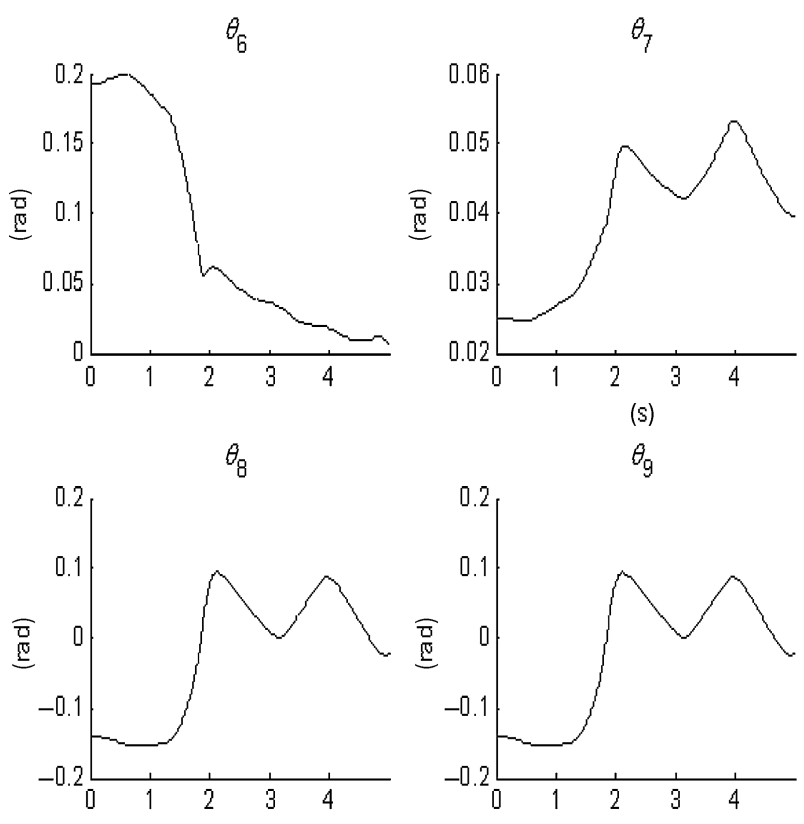

(s)

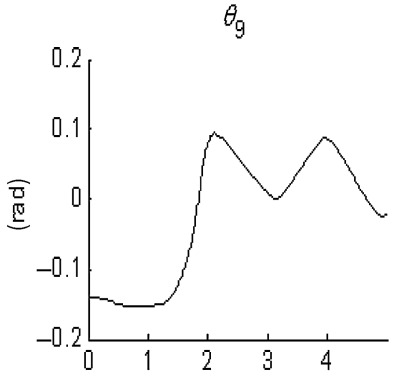

(s)

Figure 25. Joint angles of the right arm.

Figure 27. Joint angles of the left arm. 

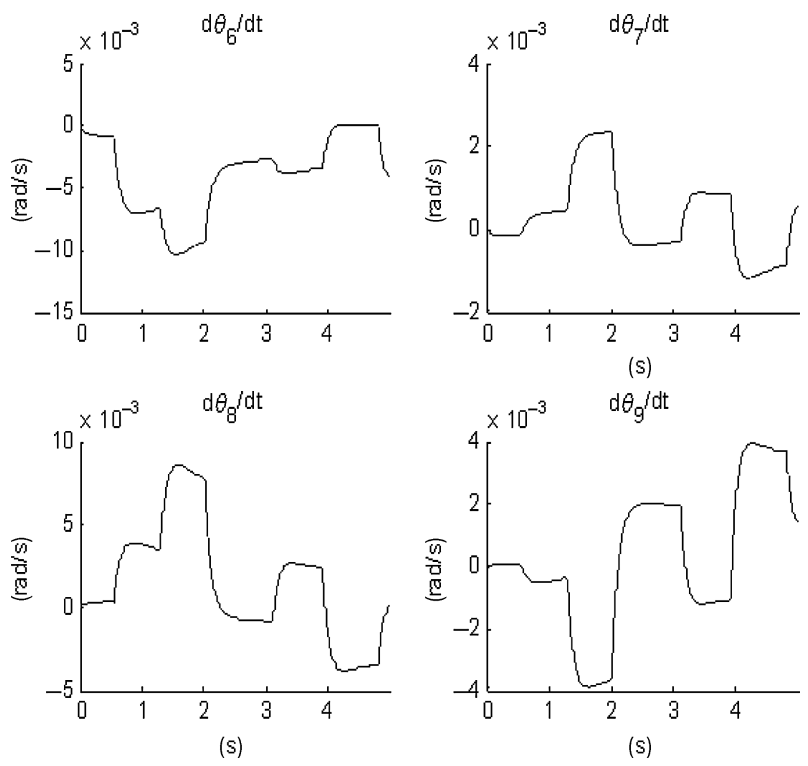

Figure 28. Joint velocities of the left arm.

establishing position and orientation of the end effector could be fulfilled.

\section{Conclusions and future works}

A control architecture for human-robot cooperation in collaborative environments has been presented. The different control loops have been analysed and simulated for the case of the humanoid robot RH-1. The posture stability has been achieved by using the model of the inverted pendulum and controlling the ankle of the robot. A following step will be the control of the system in closed loop so that it can be robust to disturbances and model mismatches. The collaborative control loop has been also analysed for the robot. A closed-chain solution for the RH-1 arms supporting an object has been proposed using the powerful instrument of the Jacobian matrix. A virtual world has been created in Simulink ${ }^{\circledR}$ to implement the kinematic model of the robot and validate the method. A study on the addition of new DOF in the robot structure is being carried out in order to achieve the requirements for holding the object and establishing position and orientation of the end effector.

\section{Acknowledgment}

This work has been supported by the CYCIT Project PI200400325 and the European Project Robot@CWE FP6-2005-IST-5, both developed within the research team Robotics Lab in the University Carlos III of Madrid.

\section{References}

Bachelier M, Chemori A, Krut SKim YD, Park IW, Yoo JK, Kim JH, 2008. A control law for human like walking biped robot SHERPA based on a control and a ballistic phase - Application on the Cart-Table Model. Proceedings of the IEEE-RAS International Conference on Humanoid Robots; 2008 pp. 132-137; Daejeon, Korea.

Fernández V, Balaguer C, Blanco D, Salichs MA, 2001. Active human-mobile manipulator cooperation through intention recognition. Proceedings of the IEEE International Conference on Robotics and Automation (ICRA'01); 2001 pp. 2668-2673; Seoul, Korea.

Green SA, Billinghurst M, Chen X, Chase JG, 2008. Humanrobot collaboration: a literature review and augmented reality approach in design. Int. J. Adv. Robot. Syst. 5(1):1-18.

Hinds PJ, Roberts TL, Jones H, 2004. Whose job is it anyway? a study of human-robot interaction in a collaborative task. Hum-Comput Interact 19:151-181.

Isidori JA, editor 1995. Nonlinear control systems. London, Great Britain: Springer-Verlag.

Kajita S, Morisawa M, Harada K, Kaneko K, Kanehiro F, Fujiwara K, Hirukawa H. 2006. Biped walking pattern generator allowing auxiliary ZMP control. Proceedings of the IEEE/RSJ International Conference on Intelligent Robots and Systems; 2006 pp:2993-2999; Beijing, (China)

Kim JY, Park IW, Oh JH, 2007. Walking control algorithm of biped humanoid robot on uneven and inclined floor. J Intell Robot Syst 48(4):457-484.

Kim YD, Park IW, Yoo JK, Kim JH, 2008. Stabilization control for humanoid robot to walk on inclined plane. Proceedings of the IEEE-RAS International Conference on Humanoid Robots; 2008 pp. 28-33; Daejeon, Korea.

Kumar RP, Yoon JW, Kim GS, 2008. Simplest dynamic walking model with toed feet. Proceedings of the IEEE-RAS International Conference on Humanoid Robots; 2008 pp. 245-250. Daejeon, Korea.

Khalil HK, editor 1999. Nonlinear systems. Upper Saddle River, New Jersey: Pearson Education.

Nakamura Y, Hanafusa H, Yoshikawa T, 1987. Task-priority based redundancy control of robot manipulators. Int J Robot Res $6(2): 3-15$.

Sciavicco L, Siciliano B, 2000 Modelling and control of robot manipulators. 2nd ed. London, Great Britain: Springer-Verlag. 

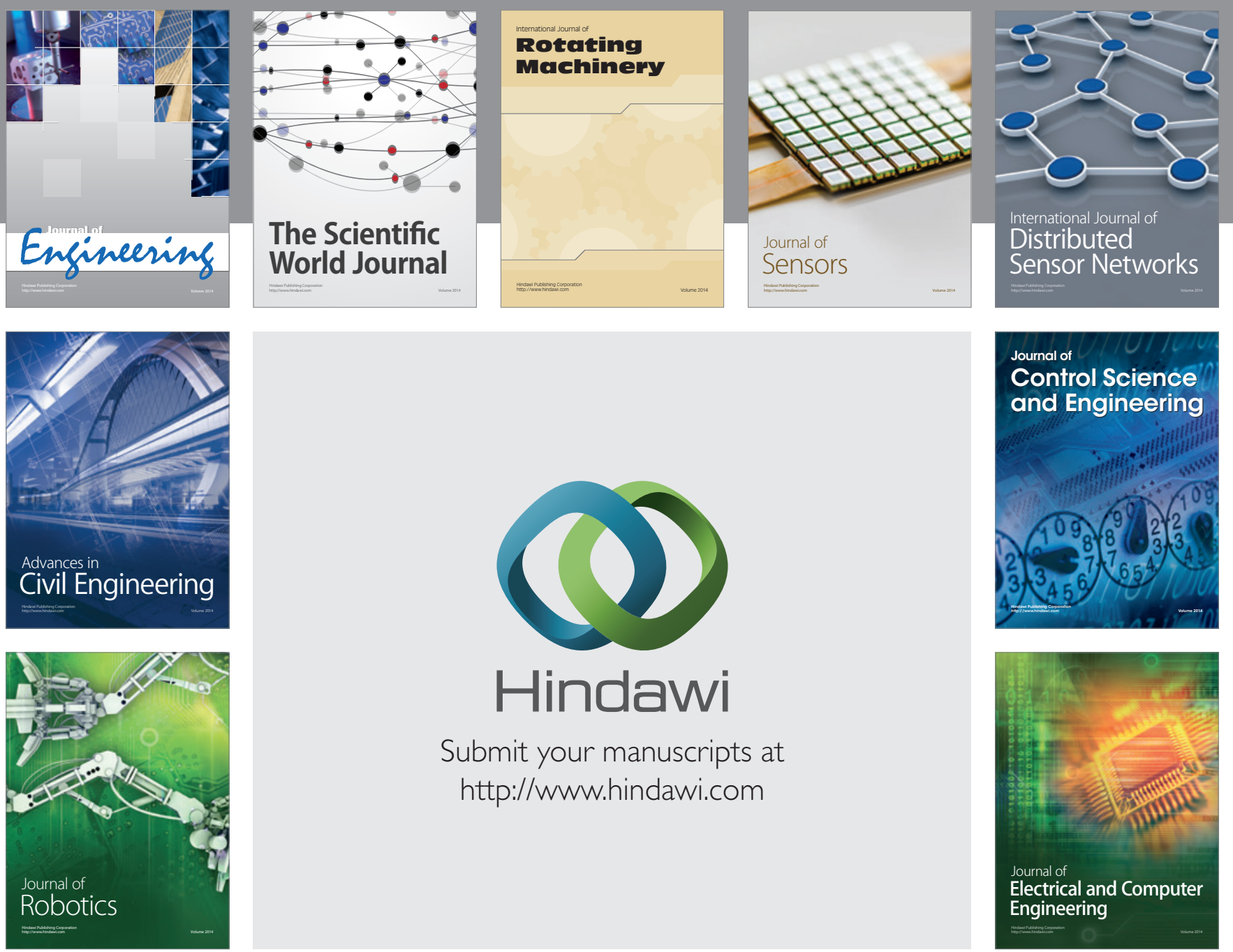

Submit your manuscripts at

http://www.hindawi.com
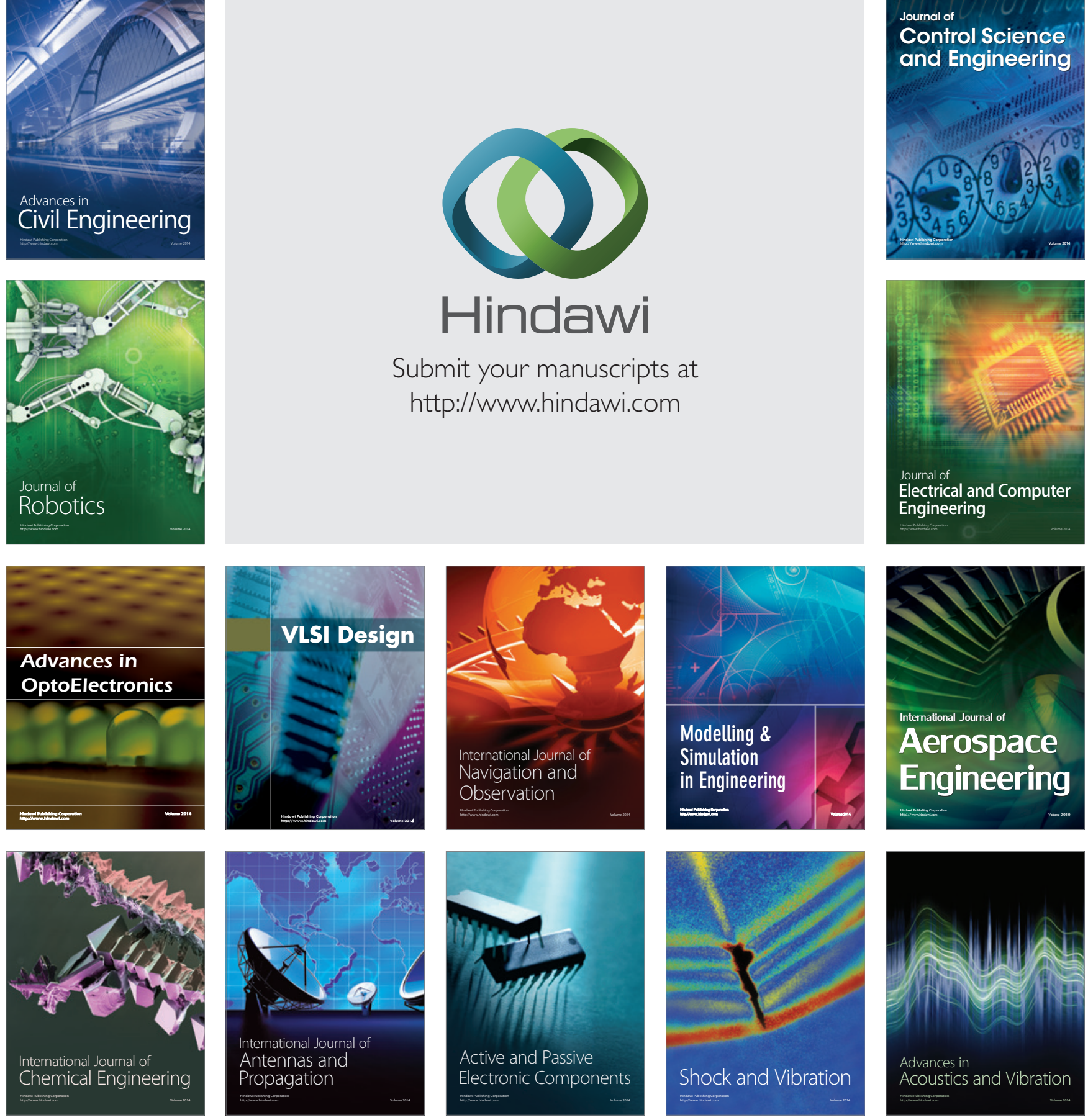